Punjab University Journal of Mathematics

(ISSN 1016-2526)

Vol. 53(1)(2021) pp. 71-82

\title{
On the Oscillatory Behavior of Some Qeneralized Differential Equation
}

\author{
Juan E. Nápoles Valdés \\ UNNE, FaCENA \\ Corrientes 3400, Argentina \\ Email: jnapoles@exa.unne.edu.ar \\ UTN-FRRE \\ Resistencia, Chaco 3500, Argentina \\ Email: jnapoles@exa.unne.edu.ar \\ Yusif S. Gasimov \\ Azerbaijan University \\ Baku, Azerbaijan \\ Institute of Mathematics and Mechanics \\ Baku, Azerbaijan \\ Institute for Physical Problems, Baku State University \\ Email: yusif.gasimov@au.edu.az \\ Aynura R. Aliyeva \\ Sumgayit State University \\ Sumgayit, Azerbaijan
}

Received: 03 July, 2020 / Accepted: 20 January, 2021 / Published online: 21 January, 2021

\begin{abstract}
In this article, using the Riccati-type transformation, we study the oscillatory nature of the solutions of the generalized differential equation and give some criteria of the Kamenev type that generalizes several well-known results on the topic.
\end{abstract}

\section{AMS (MOS) Subject Classification Codes: 34L30, 34C15}

Key Words: Generalized ordinary differential operator, oscillation, Kamenev criteria, Riccati transformation.

\section{INTRODUCTION}

Since the arbitrary order differential equations, integer or otherwise, are extensions of ordinary or partial differential equations, only in the past 40 years have been found a variety of applications and utility in various fields of science and technology (cf. [27], [30], [40] and [41]). Along with the above, several investigations have also been carried out related to the qualitative properties of its solutions (see [2], [3], [7], [8], [9], [10], [11], [12], [13], 
[15], [19], [20], [23], [28], [29], [34], [46], [47] [49], [52] and references cited therein). However, it should be noted that in those investigations, the oscillation of the solutions has not received much attention, despite the fact that it is one of the most required qualitative properties in the applications, since the existence of a certain periodic behavior, or quasiperiodically, ensures the existence of a desirable regime in the system or phenomenon studied.

Although since 2014 the definitions of the several local fractional derivatives (conformable or not) were given, all of them can be considered as particular cases (including the ordinary classic) of the definition of the Generalized Derivative, as we will see later. Between its own theoretical development and the multiplicity of applications, this field has grown rapidly in recent years. It is also known that a single definition of fractional derivative or integral does not exist, or at least is not unanimously accepted, in [6] suggests and justifies the idea of a fairly complete classification of the known operators in non-integer order Calculus. In particular, in the work [9], the reason for the appearance of new fractional operators, practically every day, linked to applications and theoretical developments is argued. On the other hand, in [4], Chapter 1, a historical tour of the classic global operators and the most recent local operators is made. Most important is the sentence in paragraph 1.4, when it concludes (in our words) "global fractional derivatives are not derivatives, local fractional derivatives are not fractional" (see also [48]). As we have said on other occasions, they are operators of different nature, which have shown their strength in many applications.

A generalized local differential operator appeared in [38] and was defined in the following way (see also [22] and [53]).

defn 1.1. Given a function $\phi:[0,+\infty) \rightarrow \mathbb{R}$. We will define the $N$-derivative of order $\alpha$ of function $\phi$ as follows

$$
N_{\Phi}^{\alpha} \phi(\tau)=\lim _{\varepsilon \rightarrow 0} \frac{\phi(\tau+\varepsilon \Phi(\tau, \alpha))-\phi(\tau)}{\varepsilon}
$$

with $\alpha \in(0,1], \tau>0$, being $\Phi(\alpha, \tau)$ the kernel of operator. Sometimes it is very useful to write this kernel $\Phi$, using the Mittag-Leffler Function $E_{v, w}($.$) , with \operatorname{Re}(v), \operatorname{Re}(w)>$ 0 . we will also consider the $k$-th term of the development of this function, designated as $E_{v, w}\left(\tau^{-\alpha}\right)_{k}$.

If we consider that $\phi$ is $N$-differentiable for certain $(0, \alpha)$, and $\lim _{\tau \rightarrow 0^{+}} N_{\Phi}^{(\alpha)} \phi(\tau)$ exists, we will put $N_{\Phi}^{(\alpha)} \phi(0)=\lim _{\tau \rightarrow 0^{+}} N_{\Phi}^{(\alpha)} \phi(\tau)$, in the case of $\phi$ differentiable, we have $N_{\Phi}^{(\alpha)} \phi(\tau)=$ $\Phi(\tau, \alpha) \phi^{\prime}(\tau)$ where $\phi^{\prime}(\tau)$ is the ordinary derivative.

It is known that this function plays a prominent role in several areas of Mathematics and, in particular, in the development of Fractional Calculus. Its classic expression is given by

$$
\mathbf{E}_{v}(z)=\sum_{k=0}^{\infty} \frac{z^{k}}{\Gamma(1+v k)}, \quad \alpha \in C, \quad \operatorname{Re}(v)>0, \quad z \in \mathbb{C},
$$

with $\Gamma$ the classic Gamma function. From seminal works of Mittag-Leffler (see [31, 32]), various studies and successive extensions of this function have appeared (see, for 
example, [14], [17, 18], [26, 44], [50]). Prabhakar introduced a new generalization $E_{v, w}^{u}(z)$ defined as ([42])

$$
E_{v, w}^{u}(z)=\sum_{k=0}^{\infty} \frac{(u)_{k}}{\Gamma(v k+w)} \frac{z^{k}}{k !}, \quad v, w, u \in C ; \quad \operatorname{Re}(v), \operatorname{Re}(w), \operatorname{Re}(u)>0, \quad z \in C,
$$

with $(u)_{k}$ the Pochhammer symbol (see [43]).

Remark 1.2. Under certain cases of the $\Phi$ kernel we can obtain some local derivatives, defined in recent years. First we note that if $\Phi(\tau, \alpha) \equiv 1$, we have the ordinary derivative. In addition if $\Phi(\tau, \alpha)=E_{1,1}\left(\tau^{-\alpha}\right)$, we have the non conformable derivative $N_{1}^{\alpha} \phi(\tau)$ defined in [21] (see also [37]); in the case that $\Phi(\tau, \alpha)=E_{1,1}\left(\tau^{1-\alpha}\right)_{1}=\tau^{1-\alpha}, \Phi(\tau, \alpha)=$ $E_{1,1}\left(\tau^{-\alpha}\right)_{1}=\tau^{\alpha}$ or $\Phi(\tau, \alpha)=E_{1,1}\left(\tau^{-\alpha}\right)_{1}=\tau^{-\alpha}$, we obtain the local derivatives of [25], [39] and [33], respectively. On the other hand, considering $\Phi(\tau, \alpha)=E_{1,1}((1-$ $\alpha) \tau)=e^{(\alpha-1) \tau}$ we obtain a conformable derivative not yet reported in the literature.

Remark 1.3. The generalized operator presented above has some qualitative differences with the ordinary derivative, which we want to point out. First, [25] presents an example (p.67) of a function $\alpha$-differentiable, which is not differentiable in the usual sense. One of the features that define a fractional derivative of a classic derivative is the "noncompliance" of the Product Rule, in [38] from Definition 1.1, a local operator is constructed that violates the product rule ( see p. 1012). We recommend section 3 of this last work ("ON THE TARASOVS AFFIRMATIONS") where we present additional details.

One of the most required properties of a derivative operator is the Chain Rule (see [38]), to calculate the derivative of compound functions, which does not exist in the case of classical fractional derivatives $N_{\Phi}^{\alpha}(f \circ g)(t)=N_{\Phi}^{\alpha} f(g(t))=f^{\prime}(g(t)) N_{\Phi}^{\alpha} g(t)$.

Next, we define the generalized integral operator that we will use in our work (see [22] and [53]):

defn 1.4. With $\alpha \in(0,1]$ and $0 \leq u \leq v$. The function $M:[u, v] \rightarrow \mathbb{R}$ we will say $\alpha$-fractional integrable on $[u, v]$, if

$$
{ }_{N} J_{u}^{\alpha} M(\chi)={ }_{N_{\Phi}} J_{u}^{\alpha} M(\chi)=\int_{u}^{\chi} \frac{M(\tau)}{\Phi(\tau, \alpha)} d \tau
$$

exists and is finite.

Remark 1.5. Taking into account the examples of kernels presented above, it is clear that we will have different integral operators. To name just one case, if $\Phi(\tau, \alpha) \equiv 1$ we will have the classic Riemann integral. This operator has also been used in the study of Integral Inequalities generalized see, for example, [1].

Remark 1.6. Obviously a local operator like the Derivative 1.1, has no direct relationship with the classic Fractional Derivatives (they are global operators), which if it has been established, see [22], the relationship between the integral operator of Definition 1.4 and fractional integral operators of the Riemann-Liouville type.

The following result is a natural extension of the classical calculus (see [22] and [53]). 
Theorem 1.7. Let $\alpha \in(0,1]$ and $\phi$ a function $N$-differentiable on $\left(\tau_{0}, \infty\right)$. The following properties are true $\left(\tau>\tau_{0}\right)$ :

a) If $\phi$ is differentiable $N_{F} J_{\tau_{0}}^{\alpha}\left(N_{\Phi}^{\alpha} \phi(\tau)\right)=\phi(\tau)-\phi\left(\tau_{0}\right)$.

b) $N_{\Phi}^{\alpha}\left(N_{F} J_{\tau_{0}}^{\alpha} \phi(\tau)\right)=\phi(\tau)$.

An important and necessary property obtained in this work is the following result

Theorem 1.8. (Integration by parts) Let $\alpha \in(0,1]$, and $u, v N$-differentiable functions on $\left(\tau_{0}, \infty\right)$. For all $\tau>\tau_{0}$ The following rule is satisfied:

$$
N_{\Phi} J_{\tau_{0}}^{\alpha}\left(\left(u N_{\Phi}^{\alpha} v\right)(\tau)\right)=\left[u v(\tau)-u v\left(\tau_{0}\right)\right]-{ }_{N_{\Phi}} J_{\tau_{0}}^{\alpha}\left(\left(v N_{\Phi}^{\alpha} u\right)(\tau)\right)
$$

The objective of this work is to investigate the oscillatory behavior of the solutions of the following non linear generalized differential equation:

$$
N^{\alpha}\left[p(\tau) N^{\alpha} y(\tau)\right]+q(\tau) N^{\alpha} y(\tau)+r(\tau) \Phi(y(\tau))=R(\tau, y(\tau)),
$$

under the following assumptions:

(I) $p \in C\left(\left[\tau_{0}, \infty\right),(0, \infty)\right)$,

(II) $q \in C\left(\left[\tau_{0}, \infty\right),[0, \infty)\right)$,

(III) $r \in C\left(\left[\tau_{0}, \infty\right), \mathbb{R}\right)$,

(IV) $0<\alpha \leq 1, f \in C(\mathbb{R}, \mathbb{R})$ such that $y \Phi(y)>0$ for $y \neq 0$, and

(V) $R(\tau, y(\tau)) \in C\left(\left[\tau_{0}, \infty\right) x \mathbb{R}, \mathbb{R}\right)$ satisfying $\frac{R(\tau, y(\tau))}{\Phi(y(\tau))} \leq s(\tau), y \neq 0$ with $s \in$ $C\left(\left[\tau_{0}, \infty\right),(0, \infty)\right)$.

We will call oscillatory any non-trivial solution of (1.4) that has infinitely large zeros; otherwise it is said to be non-oscillatory. If all the solutions of the equation (1.4) are oscillatory, then this is called oscillatory.

Models involving fractional equations and nonlinear systems play an essential role in many applications, for example, population growth, diffusion, fluid mechanics, signal theory, ..., in all these areas, as we noted before, the study of oscillating solutions, that is, the existence of a certain periodic, quasi-periodic or variable but bounded "work " regime is of vital importance in applications. It is noteworthy that, in general, they are centered on equations with the classical "global" fractional derivatives and qualitative research is almost non-existent, using local fractional derivatives (see $[35,36]$ for a tried in that direction, although in these works very different tools are used, a transformation that takes us from the non-conformable fractional equations studied to ordinary differential equations).

In this paper we study equation (1. 4 ) using the Riccati Transformation and then formulate two general oscillation criteria of Kamenev type. For this purpose the study is divided into two parts: in the first a particular case of equation (4) is studied and in the second one the general equation itself is investigated.

\section{MAIN RESUlts}

For the well known Hill equation

$$
\frac{d^{2} y}{d t^{2}}+r(\tau) y(\tau)=0
$$


Kamenev established a new oscillation criterion using an integral average method (cf. [24]), which generalizes some previous results, specifically states that if

$$
\lim _{\tau \rightarrow \infty} \sup \frac{1}{\tau^{l-1}} \int_{\tau_{0}}^{\tau}(\tau-\sigma)^{l-1} y(\sigma) d s=+\infty
$$

for $l>2$, then (2.5) is oscillatory. Based on that seminal result, we will focus our work.

2.1. A simple case. Instead of the (1. 4 ) consider the simplest case:

$$
N^{\alpha}\left[p(\tau) N^{\alpha} y(\tau)\right]+q(\tau) N^{\alpha} y(\tau)+r(\tau) y(\tau)=R(\tau, y(\tau)),
$$

subject to the conditions stated above (with $\Phi(y)=y$ ), we have the following result.

We consider a certain continuous function $M: D=\left\{(\tau, \sigma): \tau \geq \sigma \geq \tau_{0}\right\} \rightarrow \mathbb{R}$ satisfying $M(\tau, \tau)=0$ for $\tau \geq \tau_{0}, M(\tau, \sigma)>0$ for $\tau>\sigma \geq \tau_{0}$, with $\frac{\bar{\partial} M}{\partial \sigma}$ continuos and non positive function on $D_{0}=\left\{(\tau, \sigma): \tau>\sigma \geq \tau_{0}\right\}$. In addition, suppose there is a function $m: D_{0} \rightarrow \mathbb{R}$ continuous for which you have

$$
-\frac{\partial H}{\partial \sigma}(\tau, \sigma)=m(\tau, \sigma) \sqrt{M(\tau, \sigma)}
$$

for all $(\tau, \sigma) \in D_{0}$.

Theorem 2.2. Taking $M(\tau, \sigma)$ and $m(\tau, \sigma)$ defined as before. The equation (2. 7$)$ is oscillatory if the condition

$\lim _{\tau \rightarrow \infty} \sup \frac{1}{M(\tau, \sigma)} \quad N^{N} J_{\tau_{0}}^{\alpha}\left\{M(\tau, \sigma)(q(\tau)-s(\tau))-\frac{p(\sigma)}{4}\left(\frac{q(\sigma)}{p(\sigma)} \sqrt{M(\tau, \sigma)}-M(\tau, \sigma) \Phi(\sigma, \alpha)\right)^{2}\right\}=\infty$

hold.

Proof. Assume the contrary, that equation (2. 7 ) has a certain solution $y(\tau)$ with a finite number of zeros, that is, not oscillatory. From above, be $y(\tau) \neq 0$ for $\tau \geq t_{0}$. Consider the function $w(\tau)=\frac{p(\tau) N^{\alpha} y(\tau)}{y(\tau)}$, then it follows from (2.7), using the Chain Rule, the assumptions IV and V above, the following Riccati Inequality

$$
N^{\alpha} w+\frac{w^{2}}{p(\tau)}+\frac{q(\tau)}{p(\tau)} w+(q(\tau)-s(\tau)) \leq 0, \quad \tau \geq \tau_{0}
$$

Hence

$$
\begin{aligned}
& { }_{N} J_{\tau_{0}}^{\alpha}(M(\tau, \sigma)(q(\tau)-s(\tau)))(\tau) \leq \\
\leq- & { }_{N} J_{\tau_{0}}^{\alpha}\left(M(\tau, \sigma) N^{\alpha} w\right)(\tau)-{ }_{N} J_{\tau_{0}}^{\alpha}\left(M(\tau, \sigma) \frac{w^{2}}{p(\sigma)}\right)(\tau)-{ }_{N} J_{\tau_{0}}^{\alpha}\left(M(\tau, \sigma) \frac{q(\sigma) w}{p(\sigma)}\right)(\tau)
\end{aligned}
$$

Integrating by parts, rearranging and completing square we get 


$$
\begin{gathered}
{ }_{N} J_{\tau_{0}}^{\alpha}(M(\tau, \sigma)(q(\tau)-s(\tau)))(\tau) \leq M\left(\tau, \tau_{0}\right) w\left(\tau_{0}\right)- \\
-{ }_{N} J_{\tau_{0}}^{\alpha}\left[\frac{\sqrt{M(\tau, \sigma)}}{\sqrt{p(\sigma)}} w(\sigma)+\frac{\sqrt{p(\sigma)}}{2}\left(\frac{q(\sigma)}{p(\sigma)} \sqrt{M(\tau, \sigma)}-m(\tau, \sigma) \Phi(\sigma, \alpha)\right)\right]^{2}(\tau)- \\
-{ }_{N} J_{\tau_{0}}^{\alpha}\left[\frac{p(\sigma)}{4}\left(\frac{q(\sigma)}{p(\sigma)} \sqrt{M(\tau, \sigma)}-m(\tau, \sigma) \Phi(\sigma, \alpha)\right)^{2}\right](\tau) .
\end{gathered}
$$

In this way

$$
\begin{aligned}
& { }_{N} J_{\tau_{0}}^{\alpha}(M(\tau, \sigma)(q(\tau)-s(\tau))(\tau)+ \\
+ & { }_{N} J_{\tau_{0}}^{\alpha}\left[\frac{p(\sigma)}{4}\left(\frac{q(\sigma)}{p(\sigma)} \sqrt{M(\tau, \sigma)}-m(\tau, \sigma) \Phi(\sigma, \alpha)\right)^{2}\right](\tau) \leq \\
\leq & M\left(\tau, t_{0}\right) w\left(\tau_{0}\right) .
\end{aligned}
$$

From here the proof of the theorem is easily obtained, We divide by $M(\tau, \sigma)$ and take the upper limit, when $\tau \rightarrow \infty$ on both members, as the right member is bounded, we get a contradiction with the requirement (2.8).

Remark 2.3. Under the conditions $\Phi(\tau, \alpha)=E_{1,1}\left(\tau^{1-\alpha}\right)_{1}=\tau^{1-\alpha}, q \equiv 0, \Phi(y)=y$, and $R \equiv 0$ equation (1. 4 ) comes down to the equation studied in [45]. In this case Theorem 2.2 generalize the Theorem 10 given in [45, p.3]. In particular, Corollaries 12 and 13 remain valid. Of course, Theorem 14, Corollary 15, Theorem 16 and Corollary 17 can be proved in a similar way and more general results than these ones can be obtained. It is an exercise that we leave to the reader.

Remark 2.4. With $l$ an integer such that $l>2$ and using the function $M(\tau, \sigma)=(\tau-$ $\sigma)^{l-1}, \tau>s \geq \tau_{0}$, the requirements of the Theorem are fulfiled with $m(\tau, \sigma)=(l-1)(\tau-$ $\sigma)^{(l-3) / 2}$ for $\tau>\sigma \geq \tau_{0}$.

Remark 2.5. From (2. 7 ) we can obtain a damped second-order ordinary differential equation of the type $\frac{d}{d \tau}\left(p(\tau) \frac{d y}{d \tau}\right)+q(\tau) \frac{d y}{d \tau}+r(\tau) y(\tau)=R(\tau, y)$ if we use the kernel $\Phi(\tau, \alpha) \equiv 1$, so our results are consistent with those reported in the literature for this equation.

If instead of using $M(\tau, \sigma)$ as above, we directly consider $(\tau-\sigma)^{a} \sigma^{b}$ with $a \in(1, \infty)$ and $b \in[0,1)$ we can state the following result.

Theorem 2.6. The equation (2. 7 ) will be oscillatory, assuming that the conditions

$$
\begin{gathered}
\lim _{\tau \rightarrow \infty} \sup \frac{1}{\tau^{a}} \quad{ }_{N} J_{\tau_{0}}^{\alpha}(\tau-\sigma)^{a} \sigma^{b}(q(\sigma)-s(\sigma))(\tau)=\infty, \\
\lim _{\tau \rightarrow \infty} \sup \frac{1}{\tau^{a}} \quad{ }_{N} J_{\tau_{0}}^{\alpha}\left\{K(\tau, \sigma)^{2}(\tau-\sigma)^{a-2} \sigma^{b-2}\right\}(\tau)<\infty, \\
\text { are fulfilled with } K(\tau, \sigma)=\left[\frac{q(\sigma)}{p(\sigma)}(\tau-\sigma) s+a \sigma \Phi(\sigma, \alpha)-b(\tau-\sigma) \Phi(\sigma, \alpha)\right] .
\end{gathered}
$$


Proof. Proceeding as in the previous proof, after defining w in the same way, integrating and rearranging terms is obtained

$$
\begin{aligned}
& { }_{N} J_{\tau_{0}}^{\alpha}(\tau-\sigma)^{a} \sigma^{b}(q(\sigma)-s(\sigma))(\tau) \leq\left(t-\tau_{0}\right)^{a} \tau_{0}^{b} w\left(\tau_{0}\right)- \\
& -\quad{ }_{N} J_{\tau_{0}}^{\alpha}\left\{(\tau-\sigma)^{a / 2} \sigma^{b / 2} \frac{w}{\sqrt{p(\sigma)}}+\frac{\sqrt{p(\sigma)}}{2} K(\tau, \sigma)(\tau-\sigma)^{\frac{a-2}{2}} \sigma^{\frac{b-2}{2}}\right\}^{2}(\tau)- \\
& +\quad{ }_{N} J_{\tau_{0}}^{\alpha}\left\{\frac{p(\sigma)}{4} K(\tau, \sigma)^{2}(\tau-\sigma)^{a-2} \sigma^{b-2}\right\}(\tau) .
\end{aligned}
$$

Dividing by $\tau^{a}$ and taken upper limit the Theorem is obtained without difficulty.

Remark 2.7. In the case $\Phi(\tau, \alpha) \equiv 1$, the previous Theorem becomes the Theorem of [51], in particular Corollary 1 of this work is still valid. So we can state the following Kamenev generalized criteria.

Corollary 2.8. Suppose for some $a \in(1, \infty)$ and $b \in[0,1),(2.9)$ is satisfied. Then the equation (2. 7 ) is oscillatory.

2.9. A general case. Now consider the most general case, that is, the equation (1. 4 ), with $q$ a function that can be negative for arbitrarily large values of $\tau$. Thus we can state our criterion of oscillation:

Theorem 2.10. Under the conditions $p \in C\left(\left[\tau_{0}, \infty\right),(0, \infty)\right), q \in C\left(\left[\tau_{0}, \infty\right), \mathbb{R}\right), r \in$ $C\left(\left[\tau_{0}, \infty\right), \mathbb{R}\right), 0<\alpha \leq 1, f \in C(\mathbb{R}, \mathbb{R})$ such that $y \Phi(y)>0$ for $y \neq 0$ and $R(\tau, y(\tau)) \in$ $C\left(\left[\tau_{0}, \infty\right) x \mathbb{R}, \mathbb{R}\right)$ satisfying $\frac{R(\tau, y(\tau))}{\Phi(y(\tau))} \leq s(\tau), y \neq 0$ with $s \in C\left(\left[\tau_{0}, \infty\right),(0, \infty)\right)$. If, in addition, there is a function $v(\tau)>0$, with $v(\tau) \in C^{1}\left[\tau_{0}, \infty\right)$, and a constant a $\in(0, \infty)$, then the equation (2. 7 ) will be oscillatory if the following conditions are satisfied:

$$
\begin{gathered}
\lim _{\tau \rightarrow \infty} \sup \frac{1}{\tau^{a}}{ }_{N} J_{\tau_{0}}^{\alpha}\left[(\tau-\sigma)^{a}(q(\sigma)-s(\sigma)) v(\sigma)-\left\{\frac{(\tau-\sigma)^{a-2} p(\sigma)}{2 N_{F}^{\alpha} f v(\sigma)} K(\tau, \sigma)^{2}\right\}\right](\tau)=\infty \\
\text { with } K(\tau, \sigma)=\left[(\tau-\sigma) \frac{q(\sigma)}{p(\sigma)} v(\sigma)+a v(\sigma) \Phi(\sigma, \alpha)+(\tau-\sigma) N_{F}^{\alpha} v(\sigma)\right] .
\end{gathered}
$$

Proof. As in the proof of Theorem 2.2, let $y(\tau)$ be a solution with a finite number of zeros, that is, a not oscillatory solution of the equation (1.4). Then we can take $y(\tau) \neq 0$ for $\tau \geq \tau_{0}$. Let's define the function $\mathrm{w}$ as follows $w(\tau)=\frac{p(\tau) N^{\alpha} y(\tau)}{\Phi(y(\tau))}$. Then from (1.4) we have

$$
N^{\alpha} w+\frac{w^{2}}{p(\tau)} N_{F}^{\alpha} f+\frac{q(\tau)}{p(\tau)} w+(q(\tau)-s(\tau)) \leq 0, \quad t \geq t_{0} .
$$

Multiplying the above inequality by $(\tau-\sigma)^{a} v(\tau)$ integrate taking into account the Theorem 2.10 we have 


$$
\begin{aligned}
& { }_{N} J_{\tau_{0}}^{\alpha}\left[(\tau-\sigma)^{a}(q(\sigma)-s(\sigma)) v(\sigma)-\left\{\frac{(\tau-\sigma)^{a-2} p(\sigma)}{2 N_{F}^{\alpha} f v(\sigma)} K(\tau, \sigma)^{2}\right\}\right](\tau) \\
\leq & \left(\tau-\tau_{0}\right)^{a} v\left(\tau_{0}\right) w\left(\tau_{0}\right)- \\
- & { }_{N} J_{\tau_{0}}^{\alpha}\left\{\left(\sqrt{\frac{N_{F}^{\alpha} f}{p(\sigma)}}(\tau-\sigma)^{a / 2} \sqrt{v(\sigma)}\right) w+\frac{(\tau-\sigma)^{\frac{a-2}{2}} \sqrt{p(\sigma)}}{2 \sqrt{N_{F}^{\alpha} f} \sqrt{v(\sigma)}} K(\tau, \sigma)\right\}^{2}(\tau) .
\end{aligned}
$$

for all $\tau>\sigma \geq \tau_{0}$. So, we have

$$
\begin{aligned}
& { }_{N} J_{\tau_{0}}^{\alpha}\left[(\tau-\sigma)^{a} u(\sigma) v(\sigma)-\left\{\frac{(\tau-\sigma)^{a-2} p(\sigma)}{2 N_{F}^{\alpha} f v(\sigma)} K(\tau, \sigma)^{2}\right\}\right](\tau) \leq \\
\leq & \left(\tau-\tau_{0}\right)^{a} v\left(\tau_{0}\right) w\left(\tau_{0}\right) .
\end{aligned}
$$

for all $\tau>\sigma \geq \tau_{0}$. Divide the above inequality by $\tau^{a}$, we will obtain a contradiction with (2.11) if we make $\tau \rightarrow \infty$ and take an upper limit. In this way, we complete the proof.

Remark 2.11. As we said above, if we use the kernel $\Phi(\tau, \alpha) \equiv 1$, our equation (1. 4 ) is reduced to an ordinary differential equation of second order of the type $\frac{d}{d t}\left(p(\tau) \frac{d y}{d t}\right)+$ $q(\tau) \frac{d y}{d t}+r(\tau) \Phi(y(\tau))=R(\tau, y)$, therefore, the results obtained are consistent with those reported, for ordinary differential equations, in the literature.

\section{Conclusions}

In this paper various Kamenev type oscillatory results are obtained for a Generalized Differential Equation, which in the particular case turns to the classic Ordinary Differential Equations. Throughout the work the strength and extent of the obtained results, which generalize many of those reported in the literature is shown.

Acknowledgment. We would like to acknowledge the comments and suggestions of the reviewers, which allowed us to improve this work.

Author Contributions: The authors contributed equally to this work.

\section{REFERENCES}

[1] L. Aghamaliyeva, Y. Gasimov, J. E. Nápoles Valdes, On a generalization of the Wirtinger inequality and some its applications, Studia Universitatis Babe-Bolyai Mathematica, to appear.

[2] N. Aguila Camacho, M. A. Duarte Mermoud, J. A. Gallegos,Lyapunov functions for fractional order systems, Commun Nonlinear Sci Numer Simulat 19 (2014) 2951-2957.

[3] N. Aguila Camacho, M. A. Duarte Mermoud, Boundedness of the solutions for certain classes of fractional differential equations with application to adaptive systems, ISA Transactions 60 (2016) 82-88.

[4] A. Atangana, Derivative with a New Parameter Theory, Methods and Applications, Academic Press, (2016).

[5] D. Baleanu, COMMENTS ON: Ortigueira M., Martynyuk V., Fedula M., Machado J.A.T., The failure of certain fractional calculus operators in two physical models, in Fract. Calc. Appl. Anal., 22,No.2(2019) textitFract. Calc. Appl. Anal., Volume 23: Issue 1, DOI: https://doi.org/10.1515/fca-2020-0012.

[6] D. Baleanu, A. Fernandez, On Fractional Operators and Their Classifications, Mathematics,7,No.9 (2019)830; doi:10.3390/math7090830 
[7] D. Baleanu, O. G. Mustafa, R. P. Agarwal, Asymptotically linear solutions for some linear fractional differential equations, Abstract Appl. Anal. (2010), Article ID 865139.

[8] J. Baranowski, M. Zagorowska, W. Bauer, T. Dziwinski, P. Piatek, Applications of Direct Lyapunov Method in Caputo Non-Integer Order Systems, Electronika IR Electrotechnika, 21, No. 2 (2015).

[9] T. A. Burton, Fractional differential equations and Lyapunov functionals, Nonlinear Anal. 74(2011) 56485662.

[10] D. Chen, R. Zhang, X. Liu, X. Ma, Fractional order Lyapunov stability theorem and its applications in synchronization of complex dynamical networks, Commun Nonlinear Sci Numer Simulat 19 (2014) 41054121.

[11] H. Delavari, D. Baleanu, J. Sadati, Stability analysys of Caputo fractional order nonlinear systems revisited, Nonlinear Dyn, 67 (2012)2433-2439.

[12] W. Deng, Smoothness and stability of the solutions for nonlinear fractional differential equations, Nonlinear Anal. 72 (2010) 1768-1777.

[13] M. A. Duarte Mermoud, N. Aguila Camacho, J. A. Gallegos, R. Castro Linares, Using general quadratic Lyapunov functions to prove Lyapunov uniform stability for fractional order systems, Commun Nonlinear Sci Numer Simulat 22 (2015) 650-659.

[14] A. Erdelyi, F. W. Magnus, Oberhettinger, Tricomi F.G., Higher transcendental functions, New York, McGraw-Hill, 3(1955).

[15] Q. Feng, F. Meng, Interval oscillation criteria for a class of nonlinear fractional differential equations, WSEAS Transactions on Mathematics, 12, No.5 (2013) 564-571.

[16] A. Fleitas, J. A. Méndez-Bermúdez, J. E. Nápoles Valdés, J. M. Sigarreta Almira, On fractional Liénard-type systems, Revista Mexicana de Física, 65, (2019) 618-625.

[17] R. Gorenflo, A.A. Kilbas, S.V. Rogosin, On the generalised Mittag-Leffler type function, Integral Transforms Spec. Funct. 7 (1998) 215-224.

[18] R. Gorenflo, F.Mainardi, On Mittag-Leffler function in fractional evaluation processes, J. Comput. Appl.Math. 118 (2000) 283-299.

[19] P. M. Guzmán, J. E. Nápoles Valdés, A note on the oscillatory character of some non conformable generalized Liénard system, Advanced Mathematical Models \& Applications 4, No.2 (2019) 127-133

[20] P. M. Guzmán, L. M. Lugo, J. E. Nápoles Valdés, A Note on Stability of Certain Lieńard Fractional Equation, International Journal of Mathematics and Computer Science, 14,No. 2, (2019)301315

[21] P. M. Guzmán, G. Langton, L. M. Lugo, J. Medina, J. E. Nápoles Valdés, A new definition of a fractional derivative of local type, J. Math. Anal., (9:2) (2018)88-98.

[22] P. M. Guzmán, L. M. Lugo, J. E. Nápoles Valdés, M. Vivas, On a New Generalized Integral Operator and Certain Operating Properties, Axioms, 2020, No.9, 69; doi:10.3390/axioms9020069.

[23] J. B. Hu, Guo Ping Lu, Shi Bing Zhang, Ling Dong Zhao, Lyapunov stability theorem about fractional system without and with delay, Commun Nonlinear Sci Numer Simulat 20 (2015) 905-913.

[24] I. V. Kamenev, Integral criterion for oscillations of linear differential equation of second order, Mat. Zametki, 23 (1978) 249-251

[25] R. Khalil, A. Horani, M. Yousef and M. Sababheh, A new definition of fractional derivative, J. Comput. Appl. Math. 264 (2014) 65-70.

[26] A.A. Kilbas, M. Saigo, On Mittag-Leffler type function, fractional calculus operators and solution of integral equations, Integral Transforms Spec. Funct., 4 (1996) 355-370.

[27] A. Kilbas, H. Srivastava, and J. Trujillo, Theory and Applications of Fractional Differential Equations, in Math. Studies, North-Holland, New York, (2006).

[28] V. Lakshmikantham, S. Leela, M. Sambandham, Lyapunov theory for fractional differential equations, Commun. Appl. Anal. 12(2008) 365-376.

[29] Y. Li, Y. Q. Chen, I. Podlubny, Stability of fractional-order nonlinear dynamic systems: Lyapunov direct method and generalized Mittag Leffler stability, Comput. Math. Appl. 59(2010) 1810-1821.

[30] K.S. Miller, An Introduction to Fractional Calculus and Fractional Differential Equations, J. Wiley and Sons, New York, (1993).

[31] G. M. Mittag-Leffler, Sur la nouvelle fonction,C. R. Acad. Sci., Paris, 137(1903) 554-558.

[32] G. M. Mittag-Leffler, Sur la representation analytique dé une branche uniforme d'une fonction monogane, Acta Math., Paris, 29(1904) 101-181. 
[33] F. Martínez, P. O. Mohammed, J. E. Nápoles Valdés, Non Conformable Fractional Laplace Transform, Kragujevac Journal of Mathematics 46, No.3 (2022) 341354.

[34] M. Ma, D. Baleanu, Y. S. Gasimov, X. J. Yang, New results for multidimensional diffusion equations in fractal dimensional spaces, Romanin Journal of Physics, 61, No.56 (2016) 784-794

[35] J. E. Nápoles V., Oscillatory criteria for some non conformable fractional differential equation with damping, Discontinuity, Nonlinearity and Complexity, to appear.

[36] J. E. Nápoles V., On the oscillatory character of some no conformable fractional differential equation, submited.

[37] J. E. Nápoles Valdés, P. M. Guzmán, L. M. Lugo, Some new results on Nonconformable fractional calculus, Advances in Dynamical Systems and Applications, 13, No.2 (2018) 167-175.

[38] J. E. Nápoles, P. M. Guzmán, L. M. Lugo, A. Kashuri, The local non conformable derivative and Mittag Leffler function, Sigma J Eng \& Nat Sci. 38 No.2 (2020) 1007-1017

[39] J. E. Nápoles, J. M. Rodríguez, J. M. Sigarreta, On Hermite-Hadamard type inequalities for nonconformable integral operators, Symmetry 2019, No.11. 1108; doi:10.3390/sym11091108

[40] K. Oldham, J. Spanier, The Fractional Calculus. Theory and Applications of Differentiation and Integration of Arbitrary Order, Academic Press, USA, (1974).

[41] I. Podlubny, Fractional Differential Equations, Academic Press, USA, (1999).

[42] T. R. Prabhakar, A singular integral equation with a generalized Mittag-Leffler function in the kernel, Yokohama Math. J. 19 (1971) 715.

[43] E. D. Rainville, Special Functions, Macmillan, New York (1960).

[44] M. Saigo, A.A. Kilbas, On Mittag-Leffler type function and applications, Integral Transforms Spec. Funct. 7 (1998) 97-112

[45] J. Shao, Z. Zheng, Kamenev Type Oscillatory Criteria for Linear Conformable Fractional Differential Equations, Discrete Dynamics in Nature and Society, 2019, Article ID 2310185,https://doi.org/10.1155/2019/2310185

[46] H. M. Srivastava, K. M. Saad, Some new models of the time-fractional gas dynamics equation, Advanced Mathematical Models \& Applications, 3, No (1) (2018) 5-17.

[47] J. C. Trigeassou, N. Maamri, J. Sabatier, A. Oustaloup, A Lyapunov approach to the stability of fractional differential equations, Signal Process. 91 (2011) 437-445.

[48] S. Umarov, S. Steinberg, Variable order differential equations with piecewise constant order-function and diffusion with changing modes, Z. Anal. Anwend. 28 No.4 (2009) 431-450.

[49] C. Vargas De León, Volterra-type Lyapunov functions for fractional-order epidemic systems, Commun Nonlinear Sci Numer Simulat, 24 (2015) 75-85.

[50] A. Wiman, Über den fundamentalsatz in der teorie der funktionen $E_{\alpha}(z)$, Acta Math.,29(1905) 191-201.

[51] J. Yan, A note on the oscillation criterion for an equation with damped term, PAMS. 90, No.2 (1984) $277-$ 280

[52] X. J. Yang, Y. S. Gasimov, F. Gao, N. Allahverdiyeva, Travelling-wave solutions for Klein-Gordon and Helmholtz equations on cantor sets, Proceedings of the Institute of Mathematics and Mechanics, 43, No.1 (2017)123-131.

[53] D. Zhao, M. Luo, General conformable fractional derivative and its physical interpretation, Calcolo, 54: 903-917, 2017. DOI 10.1007/s10092-017-0213-8. 